physiological truth will come out of an experimental study of a pathological condition than that a fact of pathological significance will result from a purely physiological study. Every man working on a problem from either angle ought to be familiar with the other aspect. There has too long been a divorce between the two subjects, although this is tending to diminish. In recent years the Medical Research Council has endeavoured to help this situation by offering studentships to highly qualified young men to enter the field of experimental pathology as well as clinical investigation. The best qualification for such men is undoubtedly a good training in physiology, and physiologists in general would find a greater opportunity for essential discovery if they realized that a study of abnormality of function often offers the key to the elucidation of the normal process. Harvey himself did not confine his observations to one aspect of his problem. When necessary he called up evidence from every branch then known of physiology, pathology, and clinical medicine to support his argument.

\section{The Future of Medical Research}

And now, what can be said of the future? Short of a catastrophic change which may upset or even destroy national life, the future of medical discovery is bright. Past experience shows that, whatever problem concerning the body can be formulated by the human mind, there is a possibility of a solution or partial solution to it being obtained. There is thus no doubt that more and more knowledge will be obtained, giving control, both preventive and curative, of disease. The limiting factor in this progress will always be the men of genius available who make the first-class discoveries, but it is probable that with the spread of educational opportunities to poorer people more of these men will present themselves. The money available for medical research will increase, and financial obstacles will almost certainly be removed as the work develops now that the State has put its hands to the plough. No insurmountable difficulty may be expected in the direction of finance if the situation is handled wisely.

The difficulty I foresee is not that of obtaining knowledge but of its application to human needs. Curative remedies as they appear will, as in the past, undoubtedly be generally taken up by the medical profession. Much of the new knowledge to be gained, however, may concern the prevention of disease, especially the prevention of chronic and degenerative conditions, including cardiovascular disease, rheumatism, and pulmonary disease. The adoption and application of this knowledge will generally depend on the degree of education and wisdom of individuals. In some cases it will involve Government action. Without an enlightened public opinion the average individual will do nothing to save himself or herself, and the State even less. If we are to continue as a successful democracy, dependent on public opinion for action, this must be guided suitably and quickly in problems of health as in other matters.

It seems to me that one of the most important functions of this Royal College in the future will be to lead public opinion and to guide State departments, especially in matters of preventive medicine. The Royal College of Physicians is an ideal body for giving guidance and to press for action, if such be needed, in cases where scientific discovery has supplied new knowledge of importance to public health. Its independence and freedom from political relations, and the high and honourable standing of its Fellows, would give its edicts an unassailable power.

I have now completed my task of giving a bird's-eye view of the modern movement of State support for medical research. It would be idle to pretend that the State or any other body could do much more in this matter than supply facilities to assist generally, and guide the natural genius of the country in studying problems relating to health and disease. Much remains to be done to improve and develop the movement, but, bearing in mind the limitations of any such enterprise, it will probably be agreed that the entry of the State into this field of discovery has been both stimulating and fruitful.

\section{SOME ASPECTS OF RECENT WORK ON THE BACTERIOLOGY OF RHEUMATISM *}

BY

\author{
H. J. GIBSON, M.D. \\ Pathologist, Royal National Hospital for Rheumatic \\ Diseases. Bath
}

Reviews of the rheumatism problem appear with a regularity which is certainly not in keeping with the rate of advance of our knowledge of the subject, for rheumatism in its diverse forms presents an aetiological problem which is among the most difficult that medicine now faces. Consideration of its complexity suggests that entirely new conceptions of the host-parasite relationship may have to be evoked before the observed clinical, pathological, bacteriological, and serological results can be resolved into a comprehensive pattern.

For present purposes it is proposed to discuss the more recent work in its bacteriological and serological aspects, picking out certain salient and significant observations for more detailed consideration. As a starting-point the now well-known phenomenon of throat infection with Streptococcus haemolyticus followed by a latent period, which is in turn succeeded by an attack or relapse of acute rheumatism, will be considered. (Schlesinger, 1930; Collis, 1931 ; Sheldon, 1931 ; Coburn, 1931.)

Each phase has received careful study by Coburn (1932, 1935, 1936). The initial throat infection is often of very mild type, causing a slight rise of temperature, and in many cases it is overlooked by the patient. The presence or absence of tonsils does not affect the outcome, since a pharyngitis or nasopharyngitis clinically recognized as a feverish cold may be the starting-point for the rheumatic attack. Coburn and Pauli (1935d) have studied the characteristics of those strains which may be effective in producing a rheumatic relapse in susceptible subjects, contrasting them with strains which are non-effective in this respect. The effective strains were characterized by the capacity to produce strong erythrogenic toxins and haemolysins, and were in fact indistinguishable from scarlatinal strains. Those strains lacking in exotoxin production, although of human Group A and culturally similar to the effective strains, were not associated with rheumatic relapse. The toxins produced were not regarded as causative of the rheumatic lesions. On the contrary, a very low incidence of Dick-positive reactions was found among rheumatic children, and the erythrogenic toxin is

* Read in opening a discussion in the Section of Pathology, Bacteriology, and Immunology at the Annual Mecting of the British Medical Association, Plymouth, 1938. 
probably neutralized as it is produced. The significance of exotoxin production appears to be as an indication that the organism is able to elaborate other soluble antigens. It may be that some hitherto unrecognized component is the effective agent in producing the rheumatic lesions.

\section{The Latent Period}

The latent period may next be considered. This may be very short, in which case the rheumatic attack follows at once on the respiratory infection. In the majority of cases the interval is from seven to twenty-one days, after which onset of fever, with increased sedimentation rate and development of clinical signs and symptoms, ushers in the rheumatic attack.

Studies have been made of the serological changes during the latent period. The antibodies found include precipitins reacting with protein fractions and antistreptolysins. In their earlier studies Coburn and Pauli (1932c) found that in normal individuals who contracted haemolytic streptococcal infection a slight concentration of precipitin appeared about four weeks after infection in the absence of rheumatic sequelae. In cases where rheumatism followed, the precipitin reaction was more definite. Schlesinger et al. (1935) have also noted this. They considered that the antibody-producing mechanism of rheumatic patients appeared to react more strongly to acute streptococcal infections than that of the nonrheumatic. They noted that the antibody content of the patient's serum was at its height just when a relapse was to be expected. Turning next to the antihaemolysin, an antibody which is susceptible to accurate titration, we find similar results. Taking first non-rheumatic individuals, Coburn and Pauli (1932c) found that patients acutely ill with haemolytic streptococcus infection showed no change in titre but that a marked rise occurred during convalescence.

The same authors (1935f) confirmed this in rheumatic children. They showed clearly that in a group which developed rheumatic attacks the antistreptolysin titre rapidly rose to reach its maximum two to five weeks after the infection. On the other hand, failure to find a rheumatic relapse was associated with little or no rise of antistreptolysin titre even though the infecting organism possessed all the characters of an "effective" strain. They concluded that the greater the antibody response the more severe was the accompanying attack. As a corollary to this they suggested a conception of rheumatic disease as being the result of the following sequence: (1) infection with toxin-producing strains which initiates a process peculiar to rheumatic subjects; (2) antibody response, associated with (3) release of a substance, presumably from the antibody-producing tissues, which is toxic and which directly or indirectly alters mesodermal structures.

Coburn (1936) suggested that the rheumatic subject is characterized by a delayed development of antibodies. The evidence on which this conclusion is founded is not altogether clear. Thus in scarlet fever without rheumatic complication the maximum titre was found within seventeen days, while two cases of streptococcal infection with rheumatic relapse are quoted which showed a maximum on the twelfth and nineteenth days respectively.

Referring to the fact that some cases of pharyngitis are followed by rheumatism while others are not, Coburn stated :

" In both types of subjects immune responses vary widely in degree, occasionally failing to appear. When antibodies develop in the normal subject they are not associated with disease, but the development of an immune response in rheumatic subjects is frequently accompanied by the appearance of rheumatic activity."

In view of these facts, with which most workers are in agreement, the only proof that a delay in antibody response is characteristic of rheumatism would appear to be a statistical one. It must be emphasized that delay does not imply deficiency, since the response was maximal, and increased progressively, in the more severe cases, and fall of antibody did not occur till after the disease had become clinically quiescent.

\section{Persistent Infection by Haemolytic Streptococci}

The presence of antibodies to high titre in the serum of cases of acute rheumatism irrespective of a history of Strep. haemolyticus infection has been amply corroborated by Todd (1932), who was instrumental in demonstrating the antigenic properties of streptococcal haemolysin, and by Myers and Keefer (1934) ; while Hadfield, Magee, and Perry (1934) have shown that the antifibrinolysin response follows a similar course. In association with the very definite epidemiological relation between haemolytic streptococcal infection and initial attacks of acute rheumatism as shown by institutional outbreaks (Bradley, 1932; and others), this serological evidence strongly suggests that a persistent infection with that organism is an important factor. That it is operative in every case is difficult to disprove, because the period during which the organism may be found on the respiratory mucosae is known to be a short one.

Failure to elicit a history of sore throat may be explained by the mildness of the condition, which is indeed a very characteristic feature of the prodromal infection. Thus we (Gibson and Thomson, 1933) were able to secure a history of respiratory infection within the three weeks preceding the attack in only 27 per cent. of the 119 rheumatic cases investigated in Edinburgh. As illustrating how extraneous circumstances may influence such an investigation, a year later over sixty post-scarlatinal rheumatic cases were treated in the hospital in which our series was studied. Thus a similar investigation on cases from the same population in the same hospital one year later would have produced an entirely different impression. Shapiro (1935) also could find no evidence that respiratory infection preceded the rheumatic attack in any large proportion of cases. Colds, sore throats, scarlet fever, and otitis accounted for only 30 per cent. of his cases. In 45 per cent. the onset was gradual without any evidence of infection. That writer records a number of instances where injury was followed by acute rheumatism after a latent period of some days. Wilson et al. (1934) from a study of convalescent rheumatic cases obtained evidence that did not support the conception of a specific aetiological relationship between respiratory infections and rheumatic fever in children. Thus less than 10 per cent. of the rheumatic attacks were preceded within three weeks by a respiratory infection. They further reported that twothirds of the subjects experiencing rheumatic activity unassociated with respiratory infections did not exhibit a rise of antistreptolysin titre.

The failure of infections other than haemolytic streptococcal ones to activate rheumatism has been noted by Collis (1931) in the case of pneumococcal infection and Coburn and Pauli (1935b) in the case of influenza and chickenpox. Bland and Duckett Jones (1935), on the other hand, have reported pyelitis, herpes zoster, and a febrile reaction to the Schick test as being activating factors. They were also able to show repeatedly the phenomenon of latent period followed by relapse after the intravenous injection 
of T.A.B. vaccine. Operations and accidents were found by these authors to act in the same way. Thus haemolytic streptococcal infection may not be the only activating factor. Conversely, haemolytic streptococcal infection in erysipelas does not appear to be capable of initiating the rheumatic process. This is indicated by Keefer and Spink (1937), who showed that the strains responsible were mainly toxigenic, with all the other attributes of " effectiveness" in the sense of Coburn. The immune response was also shown to be efficient by the rise of antistreptolysin and antifibrinolysin in the blood, and a marked delay in the fall of the former was noted.

To sum up, epidemiological and serological findings taken together provide a striking mass of evidence in favour of the aetiological relation of the haemolytic streptococcus to acute rheumatism. Contrary evidence gathered from sporadic cases, which by their nature are not susceptible to bacteriological study in the pre-rheumatic phase, is much less convincing; but the possibility cannot be ignored that there may be a group of cases which arise without a preceding acute haemolytic streptococcal infection. Such cases may be overlooked entirely if such infections, epidemic or sporadic, are made the starting-point for the collection and study of rheumatic cases, in all of which there will naturally be serological residua in the form of specific antibodies.

\section{Theories of Aetiology}

The full interpretation of these results is admittedly impossible. -Rather they enable us to discuss hypothetical mechanisms by which the rheumatic response may be produced. Two alternative theories appear to fit the observed facts. Either the disease is an infection with Strep. haemolyticus -and this includes the possibility of chrcnic systemic infection of a type not now recognized -or some other specific rheumatic agent is primary and the streptococcus is to be regarded as merely one of a number of secondary inciting causes.

\section{Theory of Chronic Haemolytic Streptococcal Infection}

Direct evidence of this is slight. Organisms isolated from the blood, joints, and elsewhere have been mainly non-haemolytic types (Poynton and Paine, 1913 ; Clawson, 1925 ; Cecil, Nicholls, and Stainsby, 1929), and reports of such bacteriological observations are conflicting. In this connexion there are two circumstances which should be mentioned. First, the latent period allows time for the development of antibodies, and the in vivo culture medium of persisting organisms may become antagonistic to survival in their original form. The conditions are, in fact, those used artificially to enforce dissociation of other bacteria into variant forms. Secondly, in gonococcal infection of joints it is often found that the blood and joint fluid are sterile. This has been emphasized by Myers, Keefer, and Holmes (1934), who found, even in acute cases, that isolation of the organism from the joint fluid was only possible in 25 per cent. of cases. They concluded that the inflammatory reaction in cases with noninfected fluids is below the surface of the synovial membrane and in the periarticular tissues. They compared the non-infected effusion to that sometimes found in the pleura in pneumonia, the aseptic meningitis of extradural or brain abscess, and the sterile effusions in joint cavities in csteomyelitis. For these two reasons the failure to find organisms or the finding of types differing from those of the respiratory tract is not conclusive evidence against a generalized low-grade infection.
The failure to find bacterial products of primary toxicity has led to the theory of allergy, in which the specific element of the process is transferred to the tissues, the bacterial substance involved being an antigen of no primary toxicity, possibly derived from any of a number of bacterial types. The acceptance of this theory presupposes that we know all regarding the pathogenic potentialities of the streptococcus and that this knowledge will not explain how chronic infection with that organism could cause disease of the rheumatic type by the wellrecognized modes of invasion or toxin production. Such an assumption is not justified. Within the last few years we have learned of antigenic haemolysin, fibrinolysin, capsule production as a general phenomenon among haemolytic strains, the Lancefield groups, and the Griffith types, and much evidence has been given that Dick toxin is manifold in its structure and perhaps also in its works. The antibody response so characteristic and of such a marked degree and duration in acute rheumatism may simply be an indicator of the diffusion of other exoproducts possessing specific toxicity which have yet to be defined. Recent work on the multiple toxins produced by $B$. welchii and their specific effects in a variety of animal diseases is an instructive analogy.

The allergic or hyperergic variant of this theory has recently acquired support from the histological and experimental studies of Klinge (1936) and others, and merits further comment. It has been stated in its most recent form by Swift, Moen, and Hirst (1933) as follows:

"The primary haemolytic streptococcus infection is followed quickly by a state of antitoxic immunity, in contrast to a relatively delayed type-specific antibacterial immunity, and a state of bacterial hypersensitivity (hyperergy to streptococci) is concomitantly induced. With the development of partial immunity, the persisting streptococci are reduced to a state of relative avirulence for the individual, but continue to be active in the tonsils, lymph nodes, sinuses, and other tissues, where they set up focal infections, which are ideal sites for the further continuous stimulation of a hypersensitive state of the entire body. Moreover, the persistence of streptococci in these foci results in the elaboration of poisonous substances, either from the bacterial bodies or from the patient's tissues, or from both, which irritate and damage certain portions of the hypersensitive mesenchymal system."

In this form the allergic sequence resembles closely the phenomena which constitute chronic infection-for example, in syphilis or tuberculosis, where late manifestations are undoubtedly coloured by allergy. In such chronic infections the antigen circulates in the form of whole organisms capable of multiplying at the fresh sites of involvement. In rheumatism the suggestion is that bacterial products carried by the blood stream are the active agents producing similar widespread tissue changes without actual metastatic infection.

The hyperergic reaction probably has a place whatever the nature of the primary infecting agent, and is an important field of investigation which will certainly lead to a fuller understanding of the pathogenesis of the disease. The allergic conception of acute rheumatism was originally derived from the analogy between the latent period common to that disease and serum sickness. Schlesinger et al. (1935) have, however, pointed out that the same interval is noted between the initial infection and frankly septic complications such as otitis, meningitis, and adenitis, which are not regarded as allergic in any of the many. senses of that term. Acute glomerulonephritis occupies an interesting position because it follows streptococcal infection after a latent period and a high serum antistreptolysin titre is of frequent occurrence (Seegal and Lyttle, 1933 ; Cobuin, 1933). 
A final word may be said on the therapeutic evidence bearing on the relation of Strep. haemolyticus to acute rheumatism. Coburn and Pauli (1935e) found that active immunization with Dick toxin neither prevented streptococcus infection nor inhibited the development of the rheumatic process. Passive immunization with serum did not modify the rheumatic relapse and possibly increased its severity. Sulphanilamide has been shown by Swift et al. (1938) to be definitely harmful in cases of established rheumatism. Under its influence in certain cases the pulse was more rapid, pyrexia increased, fresh joints became involved, nodules appeared, and pancarditis developed. There was no evidence that antistreptolysin production was influenced, and, on the contrary, rising antibody curves were noted after administration of the drug.

Swift points out that such incidence has no real bearing on the hyperergic theory because sulphanilamide is known not to be effective in chronic infections. Eason and his co-workers $(1934,1937)$ reported favourable results after the administration of large doses of scarlatinal antitoxin in established cases, but regard the effect as partly nonspecific. Taken as a whole the therapeutic evidence does not support the streptococcal view-point, though it does not disprove it.

\section{Theory of a Specific Rheumatic Infective Agent}

The alternative of a specific rheumatic infective agent, possibly of virus type, is an almost equally satisfactory explanation of the facts as we know them. Such an agent might infect in childhood some 5 to 10 per cent. of the whole population. It would be characterized by latency, its presence only becoming clinically manifest when some incident such as infection (most frequently perhaps with haemolytic streptococcus), operation, or accident causes the balance of the symbiosis to be upset in favour of the parasite. This would account for the incidence of apparently new cases of rheumatism following outbreaks of scarlet fever or haemolytic streptococcal respiratory infection in about 10 per cent. of those attacked. That the rheumatic process may remain latent throughout life is shown by those cases which come to necropsy with scarred valves in the absence of any clinical history of rheumatism. It may be argued that the intense and prolonged antibody response to the exo-antigens of the streptococcus may be incidental and related to the inciting factor rather than to the primary cause of the disease. The rising curve of antibody titre extending over the whole period of rheumatic activity is, however, difficult to reconcile with this view. It may be noted that the persistence of the antitoxin giving rise to the Dicknegative state after scarlet fever is not considered to be due to continued activity of the streptococcus, and the same may be true of the antistreptolysin. There is, then, much to be said in favour of a specific rheumatic microorganism or virus. The work of Schlesinger, Signy, and Amies (1935), Coles (1935), and Eagles, Evans, Fisher, and Keith (1937) along these lines is interesting, and forms a valuable positive contribution to the observed phenomena of rheumatism.

Obviously no conclusion is possible at this stage. Each theory which has been put forward conflicts to a greater or less extent with the available evidence. The problem may be restated but not answered.

\section{Rheumatoid Arthritis}

The relation between acute rheumatism and rheumatoid arthritis is a subject of much controversy. Clinically there is little similarity, but on the pathological side there is a good deal of evidence that the two diseases may be aetiologically related. One may perhaps recall that erysipelas and scarlet fever are separate diseases which show little clinical relationship and yet possess in common an identical ultimate bacterial cause. For a discussion of this question the reader is referred to the review by Dawson and Tyson (1935), in which a great deal of evidence is collected. Poynton (1938) deals in a similar way with the whole group of " rheumatisms."

With regard to rheumatoid arthritis it is proposed simply to mention one aspect of recent evidence, the demonstration of serum antibodies to the haemolytic streptococcus.

Cecil, Nicholls, and Stainsby (1931) reported the isolation of haemolytic streptococci from the blood and joints of a high proportion of cases of rheumatoid arthritis, and the agglutination of the strains by the patients' sera in high dilution was shown by Nicholls and Stainsby (1931). The isolation of the organism has been confirmed by some but not by other later investigations, but the agglutination reaction has been demonstrated by practically all who have repeated it.

The titres reached, up to 1 in 10,000 or more, and the duration of the response are far in excess of those associated with acute haemolytic streptococcal infection. Dawson et al. (1932) have studied the reaction closely. They reported that the agglutinins in their thermolability and increase with the age of the subject resemble the natural antibodies of normal sera rather than the immune agglutinins following immunization or infection. They are extremely wide in their valency, and practically any haemolytic streptococcus of human group A may be used to elicit the reaction, although some strains give a higher proportion of reactors than others. The antibodies produced are thus not specific for any typical strain of rheumatic origin, as was at first suggested by Nicholls and Stainsby (1931). They were found, indeed, to react also with $\mathrm{R}$ pneumococci, although the type of agglutination was different in certain respects. The reaction showed no parallelism with the plasma and serum protein changes which are so pronounced in rheumatoid arthritis and which account for the increased sedimentation rate. They did, however, show a considerable but not complete correspondence with the precipitin content of the serum when tested with protein and carbohydrate antigens of Streptococcus haemolyticus. The antistreptolysin and antifibrinolysin of rheumatic fever are not found in rheumatoid arthritis (Stuart-Harris, 1935; and others).

Levinthal (1938) has repeated this work at the Royal National Hospital for Rheumatic Diseases, Bath. Taking a strain of haemolytic streptococcus quite at random (Group A, Griffith Type 3) from the throat of a rheumatoid arthritis patient, and examining sera "blindfold"-that is, without any knowledge of the clinical condition present-he has found agglutinin in 71.5 per cent. of cases of rhcumatoid arthritis, the reaction being strong in 51.3 per cent. His figures agree closely with those of Nicholls and Stainsby (1931), Dawson et al. (1932), Cox and Hill (1934), Blair and Hallman (1935), and McEwen et al. (1935). The reaction was negative in all but a very small proportion of other clinical types of chronic rheumatism. Of particular interest is the fact that cases of ankylosing spondylitis, by many regarded as a specialized form of rheumatoid arthritis, gave the reaction in only a very few cases. He has extended the work to a comparison of the agglutinins of joint fluid with those of blood. The observation has been made that there is a group of cases in which the test is positive in the fluid and negative in the blood serum. Out of eleven serum-negative cases eight showed positive reactions in the joint fluid.

It is argued that such joint-fluid antibodies could only have arisen from a local source-the disintegrating tissue of a joint-and that such a distribution (presence in tissue, absence in serum) is that associated with sensitization. The antigen continuously discharged from a focus of infection is unchecked by circulating antibodies and 
reaches the tissue cells, where it reacts with their antibody, leading to a local anaphylaxis, the so-called hyperergic inflammation. Levinthal suggests that the basic lesion is a debility of antibody-forming mechanism which does not permit of that prompt response necessary for effective disposal of infection. He regards the micro-organism merely as a source of antigen, the streptococcus being operative in some 90 per cent. of cases, while other bacteria may be responsible in the remainder. Dawson et al. (1932) have shown that the formation of serum agglutinins in rheumatoid arthritis is a very slow process, requiring months of active disease before high titres are reached. He and many others have observed that the titres tend to fluctuate in a remarkable way on repeated tests. Following Levinthal's views, such periods of intermission would leave the tissues vulnerable to attack.

It will be noted that the evidence is explicable by a hypothesis which is essentially similar to that of Swift in the case of rheumatic fever. It assumes that the agglutinins are true immune antibodies, and their presence is accepted as evidence of the continued presence of the streptococcus, although Dawson et al. (1932) suggest that neither assumption is fully justified on available evidence. Again, the fluctuating serum titre may recede, leaving antibody temporarily locked in the joint fluid, a circumstance which would explain the combination of "serum negative; joint fluid positive" upon which the theory is based.

Space does not permit of any further consideration of the great mass of recent work on chronic rheumatism. Hench (1938) has examined the evidence critically, and concludes as a clinical investigator that it still remains to be proved that the disease is infective in origin; as a practising physician he has committed himself with reservations to the microbic theory. This is a perfectly accurate summing-up of the present unsatisfactory position. From epidemiological considerations we may be fairly certain that acute rheumatism is infective; even this is denied us in the chronic forms. But the present revival of interest in rheumatism must in the near future produce results which will do something to remove this uncertainty.

\section{REFERENCES}

Blair, J. E., and Hallman, F. A. (1935). J. clin. Invest., 14, 505. Bland, E. F., and Jones, T. Duckett (1935). Ibid., 14, 633.

Bradley, W. H. (1932). Quart. J. Med., N.S., 1, 79.

Cecil, R. L., Nicholls, E. E., and Stainsby, W. J. (1929). J. exp. Med., 50, 617.

- (1931). Amer. J. med. Sci., 181, 12

Clawson, B. J. (1925). J. infect. Dis., 36, 444.

Coburn, A. F. (1931). The Factor of Infection in the Rheumatic State, Baltimore.

(1933). Amer. J. Dis. Child., 45, 933.

(1936). Lancet, 2, 1025.

and Pauli, R. H. (1932a). J. exp. Med., 56, 609.

- (1932b). Ibid., 56, 633.

- (1932c). Ibid., 56. 651

(1935a). Ibid., 62, 129

(1935b). Ibid., 62, 137

(1935c). Ibid., 62, 159.

(1935d). J. clin. Invest., 14, 755.

(1935e). Ibid., 14, 763 .

(1935f). Ibid., 14, 769.

(1935g). Ibid., 14, 783 .

Coles, A. C. (1935). Lancet, 2, 125

Collis, W. R. F. (1931). Ibid., 1, 1341

Cox, K. E., and Hill, D. F. (1934). Arch. intern. Med., 54, 27.

Dawson, M. H., Olmstead, M., and Boots, R. H. (1932). J. Immunol., 23, 187, 205.

and Tyson, T. L. (1935), J. Lab. clin. Med, 21,575

Eagles, G. H., Evans, P. R., Fisher, A. G. T., and Keith, J. D. (1937). Lancet, $2,421$.

Easson, J., and Carpenter, G. (1937). Quart. J. Med.. N.S., 6, 93.

- and Thomson, W. A. R. (1934). Edinb. med. J., 41, 583.
Gibson, H. J., and Thomson, W. A. R. (1933). Edinb. med. J., 40 (Med.-Chir. Soc.), 93.

Hadfield, G., Magee, V., and Perry, C. B. (1934). Lancet, 1, 834. Hench, P. S. (1938). A Survey of Chronic Rheumatic Diseases, p. 35, London.

Keefer, C. S.,. and Spink, W. W. (1937). J. clin. Invest., 16, 155,

Klinge, F. (1936). Acta Rheum., 8, No. 30, 4.

Levinthal, W. (1938). Proceedings of Rheumatism Congress, Bath (in press).

McEwen, C., Alexander, R. C., and Bunim, J. J. (1935). J. Lab. clin. Med., 21, 465.

Keefer, C. S., and Spink, W. W. (1937). J. clin. Invest., 16, 155. and Holmes, W. F. (1934). Ibid., 13, 767

Nicholls, E. E., and Stainsby, W. J. (1931). Ibid., 10, 323.

Poynton, F. J. (1938). A Survey of Chronic Rheumatic Diseases, p. 25, London.

and Paine, A. (1913). Researches on Rheumatism, London.

Schlesinger, B. (1930). Arch. Dis. Childh., 5, 411.

- Signy, A. G., and Amies, C. R. (1935). Lancet, 1, 1145.

- - and Payne, W. W. (1935). Ibid., 1, 1090.

Seegal, D., and Lyttle, J. D. (1933). Proc. Soc. exp. Biol., N.Y., $31,211$.

Shapiro, M. J. (1935). J. Lab. clin. Med., 21, 564.

Sheldon, W. (1931). Lancet, 1, 1337.

Stuart-Harris, C. H. (1935). Ibid., 2, 1456.

Swift, H. F. (1935). J. Lab. clin. Med., 21, 551.

Moen, J. K., and Hirst, G. K. (1938). J. Amer. med. Ass., $110,426$.

Todd, E. W. (1932). Brit. J. exp. Path., 13, 248.

Wilson, M. G., Wheeler, G. W., and Leask, M. M. (1934). J. clin. Invest., 14, 333.

\section{ALCOHOL INJECTION IN INOPERABLE MALIGNANT GROWTHS OF THE JAWS AND TONGUE}

\author{
BY
}

\section{WILFRED HARRIS, M.D., F.R.C.P.}

Consulting Physician, St. Mary's Hospital; Physician to the Maida Vale Hospital for Nervous Diseases

Carcinoma of the tongue and malignant growths in the facial bones, especially in the antrum, maxilla, and. nasopharynx, whether they are called sarcoma, carcinoma, or endothelioma, are in the first instance naturally the affair of the general or of the ear, nose, and throat surgeon. Sometimes one of these cases in the initial stages presents itself as a case of neuralgic pain in the face, and there may be no clear localizing signs of growth. In this way I have seen early a few cases of nasopharyngeal growths, tumours of the bones of the base of the skull, and even antral growths invading the maxilla, though the obvious evidences of antral opacity in these latter should have been sufficient to side-track such patients from a neurologist.

It is not, however, of the early diagnosis of these difficult cases that I wish to write, but rather to draw attention to the terrible pain that many of these patients suffer in the later stages, perhaps after an excision of half the tongue or of the maxilla, or after treatment of a nasopharyngeal tumour by $x$ rays, radium bomb, or radium needles. I have yet to see a nasopharyngeal malignant growth cured by surgical removal, and though radium treatment may cause temporary disappearance of the growth and great amelioration of the symptoms, yet recurrence after the lapse of a few months is the rule, and further treatment on the same lines is useless as the new growth now appears to be radium-fast.

When the surgeon in charge has decided that no further treatment can arrest the growth, it is then essential to render the remainder of the patient's life as bearable as 